Each normal $C_{n}$ image of a line meets each base $S_{n-2}$ in $n-2$ points and does not intersect the ruled variety.

The images of planes intersect $R$ in $(n+1)(n-2) / 2$ lines. The plane meets each base $S_{n-2}$ in a point, the image of which is a line meeting $n$ of the base $S_{n-2}^{\prime}$ and lying on $F_{2}$. Each base $S_{n-2}$ meets $R$ in a manifold of dimensionality $n-3$ and of order $n-1$. For $n=4$, the two-dimensional variety of order 5 has an infinite number of plane elliptic cubic curves, but the corresponding property is not true for larger values of $n$ although the intersections of each base $S_{n-2}$ and $R$ are birationally equivalent.

Cornell UNIVERSity AND

Wells College

\title{
ON THE CHARACTERISTIC ROOTS OF MATRIC POLYNOMIALS*
}

BY N. H. McCOY

1. Introduction. Unless otherwise stated, all matrices and polynomials are assumed to have coefficients in an arbitrary algebraically closed field $K$.

Let $A$ and $B$ denote square matrices of order $n$. If the characteristic roots of every polynomial $f(A, B)$ are all of the form $f(\lambda, \mu)$, where $\lambda$ and $\mu$ are characteristic roots of $A$ and $B$, respectively, then in accordance with a notation to be introduced below, we shall say that the matrices $A, B$ have property $\mathrm{I}_{n}$. By a theorem of Frobenius, $\dagger$ the matrices $A, B$ have this property if they are commutative, but this is by no means a necessary condition. The study of pairs of matrices having property $\mathrm{I}_{n}$ has been the subject of papers by Bruton, Ingraham, and Roth. $\ddagger$ However, in no case have conditions been obtained which are both necessary and sufficient for the existence of this property.

* Presented to the Society, October 26, 1935.

† G. Frobenius, Über vertauschbare Matrizen, Sitzungsberichte der Preussischen Akademie der Wissenschaften zu Berlin, 1896, pp. 601-614.

$\ddagger$ The papers by Bruton and by Ingraham have not yet been published in full but abstracts are available as follows: G. S. Bruton, Certain aspects of the theory of equations for a pair of matrices, this Bulletin, vol. 38 (1932), p. 633; M. H. Ingraham, $A$ study of certain related pairs of square matrices, this Bulletin, vol. 38 (1932), pp. 633-634. Roth's paper is On the characteristic values of the matrix $f(A, B)$, Transactions of this Society, vol. 39 (1936), pp. 234-243. 
If there exists a non-singular matrix $T$ such that $T^{-1} A T$, $T^{-1} B T$ are both in triangle form, ${ }^{*}$ we may say that $A$ and $B$ have property $\mathrm{II}_{n}$. Williamson $\dagger$ has studied the problem of determining conditions under which the matrices $A, B$ have property $\mathrm{II}_{n}$ and in particular has shown that if $A$ is non-derogatory, then property $\mathrm{I}_{n}$ implies the existence of property $\mathrm{II}_{n}$, and conversely. Under the same hypothesis on $A$, he has also exhibited other necessary and sufficient conditions for the existence of property $\mathrm{II}_{n}$.

In $\$ 2$ of the present paper we shall introduce and solve a problem somewhat more general than either of those mentioned above. As a special case we shall exhibit necessary and sufficient conditions for any finite set of matrices to have property $\mathrm{I}_{n}$ or $\mathrm{II}_{n}$. In particular, it will appear that these two properties are always equivalent. Another special case yields a perfection of Frobenius' theorem, which for the case of two matrices may be stated as follows.

$A$ necessary and sufficient condition that the matrices $A, B$ have property $\mathrm{I}_{n}$ is that for every polynomial $h(A, B)$, the matrix

$$
h(A, B)(A B-B A)
$$

be nilpotent. $\ddagger$

The condition of this theorem is clearly satisfied if $A$ and $B$ are commutative or quasi-commutative. $\S$ We shall give, in $\S 3$, a direct proof of this theorem without use of the representation theory introduced in $\$ 2$.

Finally, in $\$ 4$, we point out the connection of the present paper with the theory of Lie algebras. If the field $K$ has characteristic 0 , the equivalence of properties $I_{n}$ and $I_{n}$ is seen to be an almost immediate consequence of known theorems on Lie algebras.

* That is, all elements below the principal diagonal are zero.

$\dagger \mathrm{J}$. Williamson, The simultaneous reduction of two matrices to triangle form, American Journal of Mathematics, vol. 57 (1935), pp. 281-293.

‡ See Williamson, loc. cit., p. 292.

$\S$ The matrices $A, B$ are quasi-commutative if $A B-B A$ is not zero but is commutative with both $A$ and $B$. It follows that $A B-B A$ is necessarily nilpotent. See a previous paper, On quasi-commutative matrices, Transactions of this Society, vol. 36 (1934), pp. 327-340. 
2. The General Theorem. Let $A_{i},(i=1,2, \cdots, m)$, be given matrices of order $n$, and denote by $P$ the algebra of polynomials in these matrices. The elements of $P$ then form a representation of the algebra $P$.* Hence it is known that all elements of $P$ may, by a similarity transformation, be simultaneously reduced to the form

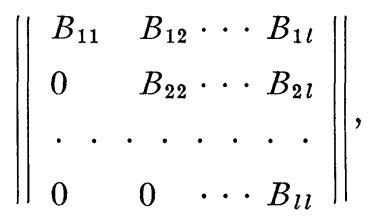

where the set $P^{(k)}$ of square matrices $B_{k k},(k=1,2, \cdots, l)$, is an irreducible representation of $P$. These irreducible components $P^{(k)}$ of $P$ are uniquely determined to within a similarity transformation and thus their orders are completely determined. The fact that $P^{(k)}$ is an irreducible representation of $P$ means essentially that $P^{(k)}$ is a simple algebra, that is, one with no proper invariant sub-algebra. We shall assume henceforth, as we may without loss of generality, that all elements of $P$ are in the form (1).

Let $Q$ denote the minimum invariant sub-algebra of $P$ containing all the elements

$$
A_{i} A_{j}-A_{j} A_{i}, \quad(i, j=1,2, \cdots, m ; i \neq j) .
$$

If now $C_{i},(i=1,2, \cdots, s)$, is a basis of $Q$, the general element of $Q$ may be written in the form

$$
Q_{x}=x_{1} C_{1}+x_{2} C_{2}+\cdots+x_{s} C_{s},
$$

where the $x_{i}$ are indeterminate elements of $K$. If $Q=0$, we shall write $Q_{x} \equiv 0$. The characteristic polynomial $\left|Q_{x}-\lambda\right|$ of $Q_{x}$ may be called the characteristic polynomial of $Q$.

Let $r$ denote a fixed integer, $0 \leqq r \leqq n$. We now define the following three properties:

$\left(\mathrm{I}_{r}\right)$. At least $r$ of the characteristic roots of every polynomial $f\left(A_{1}, A_{2}, \cdots, A_{m}\right)$ are of the form $f\left(\lambda_{1}, \lambda_{2}, \cdots, \lambda_{m}\right)$, where $\lambda_{i}$

* For proofs of the fundamental theorems on representations of algebras, see E. Noether, Hyperkomplexe Grössen und Darstellungstheorie, Mathematische Zeitschrift, vol. 30 (1929), pp. 641-692. 
is a characteristic root of $A_{i},(i=1,2, \cdots, m)$, but some polynomial has not more than $r$ characteristic roots of this form.

$\left(\mathrm{II}_{r}\right)$. Exactly $r$ of the irreducible components $P^{(k)}$ of $P$ are of order 1.

$\left(\right.$ III $\left._{r}\right)$. The characteristic polynomial of $Q$ is divisible by $\lambda^{r}$ but not by $\lambda^{r+2}$.

The principal result of this paper is the following theorem.

THEOREM 1. The properties $\mathrm{I}_{r}, \mathrm{II}_{r}$, and $\mathrm{III}_{r}$ are equivalent, $(r=0,1, \cdots, n)$.

The proof of this theorem will be made to depend upon several lemmas which we proceed to establish.

\section{LEMMA $1 . \mathrm{I}_{r} \supset \mathrm{III}_{r_{1} \geqq r} *$}

This is almost obvious. For each element of $Q$ may be expressed as a finite sum of terms of the form $g\left(A_{i} A_{j}-A_{j} A_{i}\right) h$, where $g$ and $h$ are polynomials in the $A_{i}$. Hence $\mathrm{I}_{r}$ implies that every element of $Q$ has at least $r$ zero characteristic roots. This is equivalent to property $\mathrm{III}_{r_{1} \geqq r}$. For if

$$
\left|Q_{x}-\lambda\right| \equiv(-1)^{n} \lambda^{n}+\phi_{1}(x) \lambda^{n-1}+\cdots+\phi_{n}(x),
$$

and every element of $Q$ has at least $r$ zero characteristic roots, it follows that the polynomials $\phi_{n-r+1}(x), \cdots, \phi_{n}(x)$ vanish for all choices of the $x_{i}$ in $K$. And since $K$ has an infinite number of elements, it is clear that all the coefficients in these polynomials are zero, and thus $\left|Q_{x}-\lambda\right|$ is divisible by $\lambda^{r}$.

LEMMA 2. $\mathrm{II}_{r} \supset \mathrm{I}_{r_{2} \geqq r}$.

Suppose $A_{i} \rightarrow A_{i}{ }^{(k)}$ by the correspondence $P \rightarrow P^{(k)}$. If $f\left(A_{1}, A_{2}, \cdots, A_{m}\right)$ is any polynomial, it follows from the form (1) of all matrices involved that the characteristic roots of $f\left(A_{1}, A_{2}, \cdots, A_{m}\right)$ are precisely the characteristic roots of all the matrices $f\left(A_{1}{ }^{(k)}, A_{2}{ }^{(k)}, \cdots, A_{m}{ }^{(k)}\right),(k=1,2, \cdots, l)$. If now $P^{(k)}$ is of order 1 , then $A_{i}{ }^{(k)}$ is a scalar and hence a characteristic root of $A_{i},(i=1,2, \cdots, m)$. In this case $f\left(A_{1}{ }^{(k)}, A_{2}{ }^{(k)}, \cdots, A_{m}{ }^{(k)}\right)$ is a characteristic root of $f\left(A_{1}, A_{2}, \cdots, A_{m}\right)$. Thus, if $r$ of the $P^{(k)}$ are of order 1 , at least $r$ of the characteristic roots will be of the prescribed form, which is property $I_{r_{2} \geqq r}$.

* By this notation we mean that property $\mathrm{I}_{r}$ implies the existence of property III $_{r_{1}}$ for some $r_{1} \geqq r$. 
By the homomorphic correspondence $P \rightarrow P^{(k)}$, suppose that $Q \rightarrow Q^{(k)}$. Then $Q^{(k)}$ is an invariant sub-algebra of $P^{(k)}$, and since $P^{(k)}$ is simple, must therefore be zero or $P^{(k)}$ itself. We shall now prove the following lemma.

LEMma 3. $P^{(k)}$ is of order 1 if and only if $Q^{(k)}=0,(k=1,2, \cdots, l)$.

Now $Q^{(k)}$ clearly contains the matrices $A_{i}{ }^{(k)} A_{j}{ }^{(k)}-A_{j}{ }^{(k)} A_{i}{ }^{(k)}$, $(i, j=1,2, \cdots, m)$. But $P^{(k)}$ is an algebra of polynomials in the matrices $A_{i}{ }^{(k)},(i=1,2, \cdots, m)$, with coefficients in $K$, and thus $P^{(k)}$ is commutative if $Q^{(k)}=0$. In this case $P^{(k)}$ is a representation of a commutative algebra, irreducible in an algebraically closed field. It therefore follows that $P^{(k)}$ is of order $1 .^{*}$ If, on the other hand, $P^{(k)}$ is of order 1 , it is obvious that multiplication is commutative, and, since each element of $Q^{(k)}$ is a finite sum of terms of the form $g^{(k)}\left(A_{i}{ }^{(k)} A_{j}{ }^{(k)}-A_{j}{ }^{(k)} A_{i}{ }^{(k)}\right) h^{(k)}$, where $g^{(k)}$ and $h^{(k)}$ are polynomials in the $A_{i}{ }^{(k)}$, it follows that $Q^{(k)}=0$.

We may now establish the following lemma.

\section{LEMMA 4. $\mathrm{III}_{r} \supset \mathrm{II}_{r}$.}

Let us first consider the case in which $r=n$, and assume the presence of property III $_{n}$. This means that all elements of $Q$ are nilpotent, that is, $Q$ is contained in the radical $\dagger$ of $P$. Then $Q^{(k)}$ is contained in the radical of $P^{(k)}$ and, since $P^{(k)}$ has a unit element, we must have $Q^{(k)}=0,(k=1,2, \cdots, l)$. From the preceding lemma it follows that all $P^{(k)},(k=1,2, \cdots, l)$, are of order 1 , and hence $l=n$. Thus $\mathrm{III}_{n} \supset \mathrm{II}_{n}$.

Now suppose the matrices $A_{i}$ have property III $_{r}$, where $r<n$, and thus $Q \neq 0$. By the correspondence $P \rightarrow P^{(k)}$, let $C_{i} \rightarrow C_{i}{ }^{(k)}$, $(i=1,2, \cdots, s)$. Then from (2) it follows that $Q_{x} \rightarrow Q_{x}{ }^{(k)}$, where

$$
Q_{x}^{(k)}=x_{1} C_{1}^{(k)}+x_{2} C_{2}^{(k)}+\cdots+x_{s} C_{s}^{(k)} \text {. }
$$

Now by a proper choice of the $x_{i}$ any element of $Q^{(k)}$ can be expressed in the form (3), and hence $Q^{(k)}=0$, if and only if $Q_{x}{ }^{(k)} \equiv 0$. We shall conclude the proof of the lemma by showing that precisely $r$ of the $Q_{x}^{(k)},(k=1,2, \cdots, l)$, vanish identically.

From the form (1) of all our matrices, it is clear that

$$
\left|Q_{x}-\lambda\right| \equiv \prod_{k=1}^{l}\left|Q_{x}^{(k)}-\lambda\right| \text {. }
$$

* See E. Noether, loc. cit., p. 683.

$\dagger$ Maximum nilpotent invariant sub-algebra. 
Suppose that exactly $t$ of the $Q_{x}^{(k)}$ are zero. Then we have

$$
\left|Q_{x}-\lambda\right| \equiv(-1)^{t} \lambda^{t} \Pi\left|Q_{x}^{(j)}-\lambda\right|
$$

where $j$ takes only those values for which $Q_{x}^{(j)} \not \equiv 0$, and thus $Q^{(j)}=P^{(j)}$. Hence, by the hypothesis of property $\operatorname{III}_{r}$, we have $t \leqq r$. If $t<r$, then $\Pi\left|Q_{x}^{(j)}-\lambda\right|$ must be divisible by $\lambda$, and thus $\Pi\left|Q_{x}^{(j)}\right| \equiv 0$. Hence for some $j,\left|Q_{x}^{(j)}\right| \equiv 0$. But this is impossible as $Q^{(j)}=P^{(j)}$ has a unit element, and not all elements can be singular. We must therefore have $t=r$. By Lemma 3, it follows that precisely $r$ of the $P^{(k)}$ are of order 1, and the present lemma is established.

The proof of the theorem now follows readily by formal logic. For from Lemmas 1, 2, and 4, we have

and

$$
\mathrm{II}_{r} \supset \mathrm{I}_{r_{2} \geqq r} \supset \mathrm{III}_{r_{1} \geqq r_{2}} \supset \mathrm{II}_{r_{1}} \text {, }
$$

$$
\mathrm{I}_{r} \supset \mathrm{III}_{r_{1} \geqq r} \supset \mathrm{II}_{r_{1}} \supset \mathrm{I}_{r_{2} \geqq r_{1}} \text {. }
$$

In both cases we must have $r=r_{1}=r_{2}$, and the equivalence of the three properties $\mathrm{I}_{r}, \mathrm{II}_{r}$, and $\mathrm{III}_{r}$ is at once obvious. The theorem is therefore established.

From the equivalence of properties $\mathrm{I}_{r}$ and $\mathrm{II}_{r}$ we may obtain at once the following corollary.

Corollary. Let the characteristic roots of $A_{i}$ be $\lambda_{i j}$, $(i=1,2, \cdots, m ; j=1,2, \cdots, n)$. If the matrices $A_{i}$ have property $\mathrm{I}_{r}$, it is possible to order these roots in such a way that $r$ of the characteristic roots of an arbitrary polynomial $f\left(A_{1}, A_{2}, \cdots, A_{m}\right)$ are $f\left(\lambda_{1 j}, \lambda_{2 j}, \cdots, \lambda_{m j}\right),(j=1,2, \cdots, r)$.

3. The Special Case $r=n$. The equivalence of properties $I_{n}$ and III $_{n}$ is seen to be a direct generalization of Frobenius' theorem on the characteristic roots of polynomials in commutative matrices. In the present section we shall give a direct proof of the equivalence of these two properties without introduction of property $\mathrm{II}_{n}$. If we let $N$ denote the radical of the $\operatorname{ring} P$, the theorem to be proved may be restated as follows.

Theorem 2. A necessary and sufficient condition that the matrices $A_{i},(i=1,2, \cdots, m)$, have property $\mathrm{I}_{n}$ is that the quotient ring* $P / N$ be commutative.

* The term "quotient ring" will be used for the German "Restklassenring." With this exception, the notation and terminology of this section will follow as closely as possible that of van der Waerden, Moderne Algebra, 1930 and 1931. 
The necessity of the condition is almost obvious. For property $\mathrm{I}_{n}$ implies at once that each matrix $f\left(A_{i} A_{j}-A_{j} A_{i}\right) g$, where $f$ and $g$ are arbitrary elements of $P$, is nilpotent. Hence

$$
A_{i} A_{j}-A_{j} A_{i} \equiv 0 \quad(N), \quad(i, j=1,2, \cdots, m) .
$$

We now assume that $P / N$ is commutative, and shall show that the matrices $A_{i}$ have property $\mathrm{I}_{n}$. By the homomorphic correspondence $P \rightarrow P / N$, suppose $A_{i} \rightarrow \bar{A}_{i},(i=1,2, \cdots, m)$. The elements of $K^{*}$ go over into elements of a field simply isomorphic to $K$, which we shall not distinguish from $K$ itself. Then

$$
f\left(A_{1}, A_{2}, \cdots, A_{m}\right) \rightarrow f\left(\bar{A}_{1}, \bar{A}_{2}, \cdots, \bar{A}_{m}\right) .
$$

Now let $x_{1}, x_{2}, \cdots, x_{m}$ be commutative indeterminates and consider the ring $K[x]=K\left[x_{1}, x_{2}, \cdots, x_{m}\right]$ of polynomials in the $x_{i}$. Since, by hypothesis, $\bar{A}_{i} \bar{A}_{j}=\bar{A}_{j} \bar{A}_{i}$, it follows that the correspondence

$$
g\left(x_{1}, x_{2}, \cdots, x_{m}\right) \rightarrow g\left(\bar{A}_{1}, \bar{A}_{2}, \cdots, \bar{A}_{m}\right)
$$

is a ring homomorphism between $K[x]$ and $P / N$. Hence

$$
P / N \cong K[x] / M,
$$

where $M$ is the ideal of elements $h\left(x_{1}, x_{2}, \cdots, x_{m}\right)$ in $K[x]$ such that $h\left(\bar{A}_{1}, \bar{A}_{2}, \cdots, \bar{A}_{m}\right)=0 . \dagger$

Let the minimum function of $A_{i}$ be $\phi_{i}(\lambda)$. Then $\phi_{i}\left(A_{i}\right)=0$ $=\phi_{i}\left(\bar{A}_{i}\right)$, and thus $\phi_{i}\left(x_{i}\right) \equiv 0(M)$. If $\psi_{i}\left(x_{i}\right)$ denotes the polynomial of minimum degree in $x_{i}$ alone which belongs to $M$, then $\phi_{i}\left(x_{i}\right) \equiv 0\left(\psi_{i}\left(x_{i}\right)\right)$. But $\psi_{i}\left(\bar{A}_{i}\right)=0, \quad \psi_{i}\left(A_{i}\right) \equiv 0(N)$, and thus $\left(\psi_{i}\left(A_{i}\right)\right)^{\rho}=0$. It follows that $\left[\psi_{i}\left(x_{i}\right)\right]^{\rho} \equiv 0\left(\phi_{i}\left(x_{i}\right)\right)$. Thus the roots of $\psi_{i}(\lambda)=0$ are precisely the roots of $\phi_{i}(\lambda)=0$.

By a familiar theorem, $\ddagger$ the ideal $M$ may be expressed in the form

$$
M=\left[M_{1}, M_{2}, \cdots, M_{k}\right],
$$

where the $M_{i}$ are primary ideals. Their manifolds thus consist of single points, which together form the manifold of $M$. Suppose the manifold of $M_{i}$ is $\left(\lambda_{i}{ }^{(i)}, \lambda_{2}{ }^{(i)}, \cdots, \lambda_{m}{ }^{(i)}\right),(i=1,2, \cdots, k)$.

* That is the elements of $K \cdot I$, where $I$ is the unit element of $P$.

$\dagger$ See van der Waerden, op. cit., vol. 1, p. 57.

† See van der Waerden, op. cit., vol. 2, p. 37. See also pp. 51-52, and p. 63. 
Then by the preceding paragraph, $\lambda_{j}{ }^{(i)}$ must be a characteristic root of $A_{j},(j=1,2, \cdots, m ; i=1,2, \cdots, k)$.

Now each linear expression $x_{j}-\lambda_{j}{ }^{(i)},(j=1,2, \cdots, m)$, vanishes at the point $\left(\lambda_{1}{ }^{(i)}, \lambda_{c}{ }^{(i)}, \cdots, \lambda_{m}{ }^{(i)}\right)$, and thus by Hilbert's theorem, some finite power of each of these expressions belongs to $M_{i}$. If now $f\left(x_{1}, x_{2}, \cdots, x_{m}\right)$ is any element of $K[x]$, and we expand by Taylor's theorem in powers of $x_{j}-\lambda_{j}{ }^{(i)}$, $(j=1,2, \cdots, m)$, it follows that

$$
f\left(x_{1}, x_{2}, \cdots, x_{m}\right)=f\left(\lambda_{1}^{(i)}, \lambda_{2}^{(i)}, \cdots, \lambda_{m}^{(i)}\right)+f_{i},
$$

where some finite power of $f_{i}$ belongs to $M_{i}$. Let $\alpha(\lambda)$ denote the polynomial

$$
\prod_{i=1}^{k}\left[\lambda-f\left(\lambda_{1}^{(i)}, \lambda_{2}^{(i)}, \cdots, \lambda_{m}^{(i)}\right)\right]
$$

Then for sufficiently large $\rho$, it follows from (7) that

$$
\left\{\alpha\left[f\left(x_{1}, x_{2}, \cdots, x_{m}\right)\right]\right\}^{\rho} \equiv 0 \quad(M) .
$$

Now let $f\left(A_{1}, A_{2}, \cdots, A_{m}\right)$ be any element of $P$, $f\left(\bar{A}_{1}, \bar{A}_{2}, \cdots, \bar{A}_{m}\right)$ the corresponding element of $P / N$. Then $f\left(x_{1}, x_{2}, \cdots, x_{m}\right)$ is an element of $K[x]$ which corresponds to $f\left(\bar{A}_{1}, \bar{A}_{2}, \cdots, \bar{A}_{m}\right)$ by (5). If $\alpha(\lambda)$ is defined by (8), it then follows from (9) that

$$
\left\{\alpha\left[f\left(\bar{A}_{1}, \bar{A}_{2}, \cdots, \bar{A}_{m}\right)\right]\right\}^{\rho}=0,
$$

and thus

$$
\left\{\alpha\left[f\left(A_{1}, A_{2}, \cdots, A_{m}\right)\right]\right\}^{\rho l}=0 .
$$

The distinct characteristic roots of $f\left(A_{1}, A_{2}, \cdots, A_{m}\right)$ are therefore included among the distinct roots of the equation $\alpha(\lambda)=0$, and these are all of the form $f\left(\lambda_{1}{ }^{(i)}, \lambda_{2}{ }^{(i)}, \cdots, \lambda_{m}{ }^{(i)}\right)$, where $\lambda_{j}{ }^{(i)}$ is a characteristic root of $A_{j}$. The theorem is therefore established.

4. Connection with Lie Algebras. The use of the commutator matrices $A_{i} A_{j}-A_{j} A_{i}$ suggests a connection with the theory of Lie algebras. As a matter of fact, if $K$ has characteristic 0 , we can show almost at once that property $\mathrm{II}_{n}$ is a consequence of property $\mathrm{III}_{n}$, by using known theorems on Lie algebras. 
We shall conclude with a brief statement of some facts from this point of view.*

We now assume that the field $K$ has characteristic $0 . \dagger$ A Lie algebra of matrices over $K$ is a linear space $L$ which is closed under the commutator operation, $[A, B]=A B-B A$, where $A$ and $B$ are any elements of $L$. The linear space generated by the commutators of $L$ is itself a Lie algebra, called the first derived algebra of $L$, and denoted by $L^{\prime}=[L, L]$. Then $L^{(2)}=\left[L^{\prime}, L^{\prime}\right]$, and so on. If there exists an integer $s$ such that $L^{(s)}=0$, then $L$ is said to be solvable. The $n$th power of $L$ may be defined by induction as $L^{n}=\left[L, L^{n-1}\right]$. If, for some finite $n, L^{n}=0, L$ is said to be nilpotent. Evidently $L$ is solvable if it is nilpotent.

Now let $A_{i},(i=1,2, \cdots, m)$, be given matrices of order $n$, and close the linear space generated by the $A_{i}$ with respect to the commutator operation, thus leading to a Lie algebra defined by the $A_{i}$. The elements of $L^{\prime}$ are all of the form $f g-g f$, where $f$ and $g$ are polynomials in the $A_{i}$. But in the notation of $\S 2$, multiplication is commutative $\bmod Q$, and thus all elements of $L^{\prime}$ belong to $Q$. Hence under hypothesis III $_{n}$, all elements of $L^{\prime}$ have $\lambda^{n}$ as characteristic function. It then follows by a generalization of Engel's theorem that $L^{\prime}$ is nilpotent. Hence $L$ is solvable and by Lie's theorem all elements of $L$ may be simultaneously transformed to triangle form, which is property $\mathrm{II}_{n}$.

These considerations enable us to state the following theorem. $\ddagger$

THEOREM 3. If the underlying field $K$ has characteristic 0 , $a$ necessary and sufficient condition that the matrices $A_{i}$, $(i=1,2, \cdots, m)$, have property $\mathrm{I}_{n}$ is that the Lie algebra $L$ defined by these matrices be solvable.

\section{Smith College}

* For proofs and references, see N. Jacobson, Rational methods in the theory of Lie algebras, Annals of Mathematics, vol. 36 (1935), pp. 875-885.

$\dagger$ Jacobson has pointed out to me that the Lie theorem does not hold for Lie algebras over a field of characteristic $p$. Hence this is a necessary restriction.

$\ddagger$ In this connection see a paper by W. E. Roth, On $k$-commutative matrices, Abstract No. 40-11-349. Roth considered a weaker condition than that $L^{\prime}$ be nilpotent, and discussed some of its consequences. 